\title{
Flora vascular de un remanente de bosque esclerófilo mediterráneo costero: Estación de Biología Terrestre de Hualpén, Región del Biobío, Chile
}

\author{
Vascular flora of a remnant of coastal Mediterranean Sclerophyll forest: Hualpén \\ Terrestrial Biology Station, Biobío Region, Chile
}

\author{
María Moreno-Chacón ${ }^{1}$, Daniela Mardones ${ }^{2}$, Nataly Viveros ${ }^{1 *}$, Karina Madriaza ${ }^{1}$, Fernando \\ Carrasco-Urra ${ }^{1}$, Alicia Marticorena ${ }^{1}$, Carlos Baeza ${ }^{1}$, Roberto Rodríguez ${ }^{1}$ \& Alfredo Saldaña ${ }^{1}$ \\ 1Departamento de Botánica, Facultad de Ciencias Naturales y Oceanográficas, Universidad de Concepción, Casilla 160-C, \\ Concepción, Chile. \\ ${ }^{2}$ Departamento de Ciencias Ecológicas, Facultad de Ciencias, Universidad de Chile. \\ *nviverosm@udec.cl
}

\begin{abstract}
RESUMEN
Los remanentes de los bosques esclerófilos mediterráneos costeros en Chile tienen una baja representatividad en el Sistema Nacional de Áreas Silvestres Protegidas, a pesar de su alto endemismo y riqueza de especies. En la Región del Biobío, la Estación de Biología Terrestre de Hualpén (EBT) conserva un remanente de este tipo de bosque, para el cual no existen revisiones que describan su flora, lo que dificulta la formulación y el desarrollo de estrategias para su protección y conservación. Este trabajo tiene como objetivos: a) describir la composición taxonómica de las plantas vasculares de la ETB y b) entregar información sobre su origen geográfico, hábito y estado de conservación. El listado florístico obtenido incluye tanto las especies determinadas a través del muestreo sistemático en el área de estudio durante un año, así como las especies de la EBT ingresadas en la colección del Herbario de la Universidad de Concepción. Se determinaron 294 especies de plantas vasculares de las cuales 71 son endémicas, 124 son nativas y 99 son introducidas. Desde el punto de vista florístico, la familia Asteraceae fue la má diversa con 36 especies, seguidas de Poaceae con 32 especies y Fabaceae con 18 especies. Las hierbas fueron el hábito de crecimiento más frecuente $(77,2 \%)$, seguida de las categorías arbusto (13,9\%), árbol $(7,5 \%)$ y subarbustos $(1,4 \%)$. Diecinueve especies endémicas y nativas han sido clasificadas en alguna categoría de conservación en Chile continental, destacando Gomortega keule y Pitavia punctata, ambas clasificadas como especies En peligro, y el helecho Asplenium trilobum catalogado bajo la categoría Preocupación menor en Chile y Vulnerable en la Región del Biobío. A pesar de la reducida superficie protegida en la EBT (70 ha), el lugar cuenta con una alta riqueza de especies nativas si se la compara con datos obtenidos en otros estudios de flora en sitios de Chile central.
\end{abstract}

Palabras clave: Área complementaria, Gomortega keule, Pitavia punctata, Península de Hualpén.

\begin{abstract}
The remnants of coastal Mediterranean sclerophyll forests in Chile have a low representativeness in the National System of Protected Wild Areas despite their high endemism and species richness. In the Biobío Region, the Hualpén Terrestrial Biology Station (EBT) protects a remnant of this type of forest, for which there are no revisions describing its flora, which makes it difficult to formulate and develop strategies for its protection and conservation. This work aims are: a) to describe the taxonomic composition of vascular plants of the ETB and b) to provide information on their geographical origin, habit and conservation status. The resulting floristic list includes species identified through systematic sampling in the study area for one year, as well as EBT species stored in the collection of the Universidad de Concepción Herbarium. We identified 292 species of vascular plants, where 71 species are endemic, 124 are native, 99 are alien species. From the floristic point of view the Asteraceae family was the most diverse with 36 species, followed by Poaceae with 32 species and Fabaceae with 18 species. Herbs were the most frequent growth habit (77.2\%), followed by shrub (13.9\%), tree (7.5\%) and sub-shrub $(1.4 \%)$. Nineteen endemic and native species have been classified in some category of conservation in continental Chile, standing out Gomortega keule and Pitavia punctata both classified as endangered species, and the fern Asplenium trilobum classified as a least concern in Chile and vulnerable in the Biobío Region. Despite of the reduced protected area in the EBT (70 ha), the site has a high species richness of native species when compared with data obtained from other studies of flora in sites of central Chile.
\end{abstract}

KeYwords: Complementary area, Gomortega keule, Pitavia punctata, Hualpén Peninsula. 


\section{INTRODUCCIÓN}

Actualmente el bosque esclerófilo mediterráneo costero de Chile continental está representado por fragmentos de vegetación (distribuidos entre los $31^{\circ} 52^{\prime} \mathrm{S}$ y los $37^{\circ} 20^{\prime} \mathrm{S}$ ), que albergan una alta riqueza de especies y un alto grado de endemismo (Armesto et al. 1994, Luebert \& Pliscoff 2006). Se considera que estos remanentes de bosque costero fueron un refugio para la diversidad durante el Último Máximo Glacial (Villagrán \& Armesto 2005), lo que explicaría la alta riqueza de especies que lo caracteriza. En este tipo de bosque encontramos especies cuyas poblaciones, restringidas a la zona litoral, presentan una distribución geográfica discontinua, como por ejemplo Gomortega keule (Molina) Baillon (Gomortegaceae) y Pitavia punctata Molina (Rutaceae) (Smith \& Armesto 2002), ambas en peligro de extinción, de manera que estos remanentes de vegetación son particularmente relevantes desde el punto de vista de la conservación de la flora nativa y endémica de Chile. Sin embargo, y aun cuando se reconoce la importancia de proteger y conservar este tipo de bosque en Chile (Schulmeyer 1978, San Martín \& Donoso 1995, Armesto et al. 1998, Smith-Ramírez 2004), se encuentran pobremente representados en el Sistema Nacional de Áreas Protegidas por el Estado (SNASPE) (Luebert \& Pliscoff 2006).

En la Región del Biobío existe una superficie remanente del denominado bosque esclerófilo mediterráneo costero de Lithrea caustica y Azara integrifolia (Luebert \& Pliscoff 2006) (Fig. 1b), que comprende una vegetación boscosa de tipo esclerófila dominada por Lithrea caustica (Molina) Hook. \& Arn. (Anacardiaceae), Cryptocarya alba (Molina) Looser (Lauraceae) y Azara integrifolia Ruiz \& Pav. (Salicaceae), con algunos elementos florísticos característicos del bosque maulino (e.g. Persea lingue (Ruiz \& Pav.) Nees (Lauraceae), Gevuina avellana Molina (Proteaceae), Lapageria rosea Ruiz \& Pav. (Philesiaceae) y Escallonia pulverulenta Ruiz \& Pav. (Escalloniaceae) (Bustamante et al. 2005). El área en la que se distribuye este tipo de bosque, al igual que el resto de los bosques costeros, ha sufrido un gran deterioro y degradación principalmente de origen antrópico (Aguayo et al. 2009, Miranda et al. 2015). Actualmente, presenta una superficie remanente de $1.993 \mathrm{~km}^{2}$, de los cuales sólo el $0,1 \%$ tiene representatividad en el SNASPE (Luebert \& Pliscoff 2006). A lo anterior se agrega que en la Región del Biobío la situación se agrava ya que entre los años 1986 y 2011 se perdió un $16,3 \%$ de la superficie remanente del bosque nativo producto de la sustitución de éste por plantaciones forestales de especies exóticas (Heilmayr et al. 2016). En este escenario de vulnerabilidad, las áreas complementarias al SNASPE, que incluyen santuarios de la naturaleza, áreas de protección, lugares de interés científico y reservas genéticas (CONAF-CONAMA 1997) han contribuido a resguardar la biodiversidad de los bosques esclerófilos mediterráneo-costeros de la Región del Biobío (Ibarra-Vidal et al. 2005). Un ejemplo emblemático de este tipo de sitios en la región es el Santuario de la Naturaleza de la Península de Hualpén, creado en 1976 por medio del Decreto $\mathrm{N}^{\circ}$ 556 del Ministerio de Educación, de acuerdo a la Ley $\mathrm{N}^{\circ}$ 17.288, a partir de terrenos privados que comprendían toda la Península de Hualpén, con el objetivo de resguardar el patrimonio natural que incluye el remanente de bosque nativo, el entorno biogeográfico costero fluvial (FPA 2016) y proteger las aves de los humedales (Quintana 1993). En las últimas décadas, el santuario ha estado sometido a una fuerte presión antrópica debido a las visitas masivas de turistas, al pastoreo bovino y al cambio en el uso de suelo de la matriz circundante, donde la vegetación original ha sido reemplazada por especies de árboles exóticos de interés silvicultural (e.g. Eucalyptus globulus Labill. y Pinus radiata D. Don.). A pesar de lo anterior, el santuario aún conserva fragmentos del bosque esclerófilo mediterráneo costero con remanentes de la vegetación original de la zona (Polyméris 1995).

La Estación de Biología Terrestre (EBT) se ubica en uno de los fragmentos del bosque esclerófilo costero de la Península de Hualpén, cercano a la desembocadura del río Biobío ( $36^{\circ} 47^{\prime} \mathrm{S}-73^{\circ} 10^{\prime} \mathrm{O}$, Fig. 1a y 1b). El área fue cedida en comodato por la Gobernación de Talcahuano a la Universidad de Concepción y ha sido protegida de la actividad antrópica desde el año 1970, con el fin de conservar la flora, la fauna y los servicios ecosistémicos del bosque costero, así como para permitir el desarrollo de investigaciones, docencia y actividades asociadas con la difusión de la ciencia. A pesar de la importancia de la zona biogeográfica en la que se encuentra la EBT y del contexto espacial en la que se ubica en el paisaje (i.e. zona de gran influencia antrópica), no existe una revisión actualizada que permita conocer la riqueza y la composición de su flora vascular, a excepción de una tesis de pregrado (Carrasco 1976) con escasos ejemplares y de difícil acceso, por lo que el presente estudio presenta el listado florístico más actualizado y el único publicado. Este estudio tiene como propósito contribuir al conocimiento de la flora de los remanentes del bosque costero de la Región del Biobío. Para ello se propone: a) describir la composición taxonómica de las plantas vasculares de la ETB y b) entregar información sobre el origen geográfico, hábito y el estado de conservación actualizado de las especies presentes en el área de estudio. Esta información, además de ser un aporte al conocimiento de la flora de la región, podría ser útil para el desarrollo de estrategias de protección para este remanente de bosque esclerófilo mediterráneo costero. 


\section{MATERIALES Y MÉTODOS}

Área de ESTUdio

La Estación de Biología Terrestre (36 $46^{\circ} 7^{\prime} \mathrm{S}$ y $\left.73^{\circ} 10^{\prime} \mathrm{O}\right)$ se encuentra ubicada en la Península de Hualpén cerca de la desembocadura del río Biobío (Fig. 1a y 1b) y abarca un área continua de 70 ha. El clima descrito para la península es de tipo templado, submediterráneo, con influencia oceánica. La precipitación media anual alcanza $1.100 \mathrm{~mm}$ mientras que la temperatura media anual es de $12,7^{\circ} \mathrm{C}$ (Luebert \& Pliscoff 2006). La vegetación de la ETB corresponde a un bosque esclerófilo mediterráneo costero de Lithrea caustica y Azara integrifolia con algunos elementos florísticos característicos del bosque maulino (Luebert \& Pliscoff 2006), pero sin representantes del género Nothofagus como ocurre en otros bosques costeros de la Región del Biobío (Polyméris 1995).

\section{MUESTREO Y DETERMINACIÓN DE LAS ESPECIES}

El área de estudio se visitó cada quince días, entre noviembre de 2015 y noviembre de 2016, logrando cubrir cerca del 80 $\%$ de la superficie de la ETB. En cada visita se realizó un rastreo mediante recorrido libre aleatorio, abarcando una superficie de observación de entre cuatro y seis hectáreas.
Todos los puntos de recolección fueron visitados de forma sistemática en varias ocasiones durante el muestreo para constatar la germinación y antesis de ejemplares que no fueron observados, recolectados o registrados fotográficamente con anterioridad. Los ejemplares recolectados y fotografiados fueron georreferenciados en grados sexagesimales utilizando un Sistema de Posicionamiento Global (GARMIN MODELO GPSMAP 62sc).

La determinación de los ejemplares recolectados se realizó hasta el nivel de especie y en algunos casos hasta subespecie o variedad, utilizando diversas fuentes bibliográficas (Muñoz-Schick 1980, Marticorena \& Rodríguez 1995, 2001, 2003, 2005, 2011, Matthei 1995, Hoffmann 1998, Rodríguez \& Dellarosa 1998, Donoso 2005, 2009, Teillier et al. 2005, 2014, Rodríguez et al. 2005, Zuloaga et al. 2008, Rodríguez et al. 2009, Quiroz et al. 2009, Marticorena et al. 2010, Novoa 2013, Novoa et al. 2015, Teillier \& Escobar 2013, 2014, Fuentes et al. 2014, Riedemann et al. 2014), además de la comparación con ejemplares depositados en el Herbario de la Universidad de Concepción (CONC) y la determinación de ejemplares por expertos botánicos pertenecientes al Departamento de Botánica de la Universidad de Concepción.
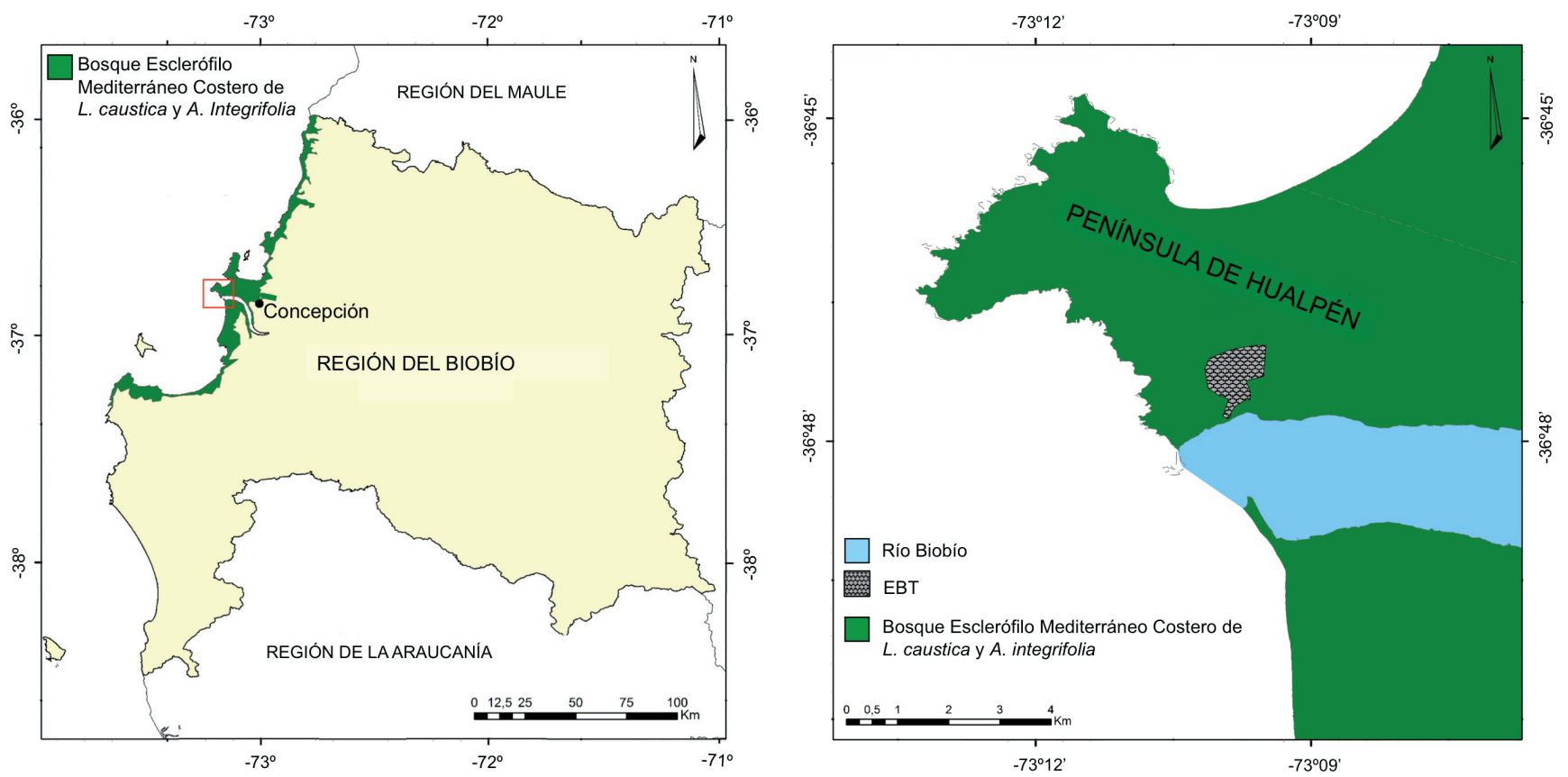

Figura 1. a) Distribución potencial del piso de vegetación del bosque esclerófilo mediterráneo costero de Lithrea caustica y Azara integrifolia (sensu Luebert \& Pliscoff 2006) en la Región del Biobío b) Ubicación de la Estación de Biología Terrestre de Hualpén. / a) Potential distribution of the vegetation belts of coastal Mediterranean sclerophyll forest of Lithrea caustica y Azara integrifolia (sensu Luebert \& Pliscoff 2006) in the Biobío Region. b) Location of the Hualpén Terrestrial Biology Station. 
Para la nomenclatura de las especies se siguieron los criterios utilizados en el Catálogo de Plantas Vasculares de la Flora del Cono Sur (Zuloaga et al. 2008) y sus actualizaciones posteriores (http://www2.darwin.edu.ar/ Proyectos/FloraArgentina/fa.htm, consultada hasta abril de 2017). Los ejemplares recolectados fueron herborizados, determinados y depositados en el CONC. El listado florístico de especies para la EBT incluye tanto las especies recolectadas, observadas y fotografiadas en terreno por los autores, así como aquellas provenientes del área de estudio que fueron depositadas en CONC con anterioridad a este estudio.

Al listado de especies ordenadas por clases, familias y géneros (Tabla 4) se incorporó información sobre el hábito, el origen geográfico y el estado de conservación. Para los hábitos se consideraron cuatro categorías principales: árbol, arbusto, subarbusto, hierba perenne y hierba anual, además de cinco subcategorías que se incluyen: epífitas, parásitas, apoyantes, acuáticas y trepadora (Zuloaga et al. 2008). También se recopiló información de diversas fuentes bibliográficas para clasificar las especies de acuerdo a su origen geográfico entre nativas, endémicas e introducidas en Chile (Muñoz-Schick 1980, Marticorena 1990, Mösbach 1992, Arroyo et al. 2005, Rodríguez et al. 2005, Teillier et al. 2005, García \& Ormazábal 2008, Rodríguez et al. 2008a, 2008b, Zuloaga et al. 2008, Gómez et al. 2009, Finot et al. 2009, Rodríguez et al. 2009, Garcia 2010, Marticorena et al. 2010, Fuentes et al. 2013, Novoa 2013, Teillier \& Escobar
2013, Freire et al. 2014, Fuentes et al. 2014, Niemeyer 2014, Riedemann et al. 2014, Teillier et al. 2014, Novoa et al. 2015).

Finalmente, para las especies nativas y endémicas se registró su estado de conservación de acuerdo a la información actualizada en los diferentes procesos de clasificación de especies disponibles en la página web del Ministerio del Medio Ambiente (MMA) (http://www. mma.gob.cl/clasificacionespecies/listado-especies-nativassegun-estado-2014.htm revisado en marzo de 2017).

\section{RESULTADOS}

\section{COMPOSICIÓN TAXONÓMICA}

Se determinaron 294 especies de plantas vasculares para la EBT, distribuidas en 90 familias y 222 géneros (Tabla 1). Las familias Asteraceae, Poaceae y Fabaceae tuvieron mayor representatividad con 36, 32 y 18 especies, respectivamente. La clase Magnoliopsida representa con 207 especies el 70,4\% de la riqueza presente en la EBT, las que se distribuyen en 66 familias y 163 géneros, mientras que la clase Liliopsida alcanza el $22,8 \%$ de la riqueza con 67 especies incluidas en 15 familias y 47 géneros (Tabla 1). Por su parte, la clase Polypodiopsida es el grupo con menor representatividad en el área de estudio con 9 familias y 12 géneros, alcanzando el $6,8 \%$ de las especies descritas (Tabla 1).

TABLA 1. Riqueza de familias, géneros y especies de plantas vasculares presentes en la Estación de Biología Terrestre de Hualpén. / Richness of families, genera and species of vascular plants present in the Hualpén Terrestrial Biology Station.

\begin{tabular}{lccc}
\hline Clase & FAmilias & Géneros & EsPecies \\
\hline Polypodiopsida & 9 & 12 & 20 \\
Magnoliopsida & 66 & 163 & 207 \\
Liliopsida & 15 & 47 & 67 \\
\hline TotaL & 90 & 222 & 294 \\
\hline
\end{tabular}

TABLA 2. Número de especies para los cuatro principales hábitos presentes en la Estación de Biología Terrestre de Hualpén, considerando el origen geográfico (endémica, nativa, introducida). / Number of species in the four main habits present at the Hualpén Terrestrial Biology Station considering the geographic origin (endemic, native, alien).

\begin{tabular}{lcccc}
\hline HÁbito & ENDÉmicA & NAtiva & InTRoducidA & TotaL \\
\hline Árbol & 9 & 12 & 1 & 22 \\
Arbusto & 18 & 16 & 7 & 41 \\
Hierba & 42 & 94 & 91 & 227 \\
Subarbusto & 2 & 2 & 0 & 4 \\
\hline Total & 71 & 124 & 99 & 294 \\
\hline
\end{tabular}




\section{HÁBITOS}

Entre los cuatro hábitos considerados en este estudio, las hierbas con 227 especies representa el 77,2\% de las especies identificadas en la EBT (Tabla 2) e incluyen las subcategorías de crecimiento anual, bienal, perenne, acuático, parásito, trepador y palustre (detalles ver Tabla 4). Los arbustos alcanzan un 13,9\% de representatividad con 41 especies (Tabla 2), algunas de las cuales se encuentran en las subcategorías de crecimiento trepadora y parásita (Tabla 4). Finalmente, los árboles y subarbustos son los hábitos con menor número de especies, alcanzando un $7,5 \%$ y un $1,4 \%$ del total de especies respectivamente.

\section{ORIGEN GEOGRÁFICO}

En relación al origen geográfico, el 42,1 \% de las especies presentes en la EBT corresponde a especies nativas, el 24,1\% endémicas y el 33,7 \% a introducidas (Tabla 2). Las familias con mayor número de especies nativas son Asteraceae con 13 especies, además de Cyperaceae, Myrtaceae y Violaceae con cinco especies cada una. Las familias Poaceae y Asteraceae son las que presentan el mayor número de endemismos con nueve y siete especies, respectivamente. Por su parte, las familias Poaceae, Asteraceae y Fabaceae presentan una alta representatividad de especies introducidas con 19, 16 y 12 representantes respectivamente (ver detalles Tabla 4).

Considerando el hábito, encontramos que los árboles en su mayoría corresponden a especies endémicas (9) y nativas (12), con una especie introducida (Salix babylonica L., Salicaceae). Por su parte los arbustos tienen un alto número de representantes endémicos (18) y nativos (16), aunque también se encontraron especies introducidas (ver detalles Tabla 4). Las hierbas representan el $91 \%$ de las especies introducidas identificadas en la EBT (Tabla 2). En el caso de los subarbustos, todos los representantes son nativos o endémicos de Chile.

ESTADO DE CONSERVACIÓN

De las 195 especies endémicas y nativas presentes en la EBT, solo 19 han sido incluidas en alguna categoría de conservación en Chile Continental. Dentro de este grupo de especies destacan Gomortega keule y Pitavia punctata ambas en la categoría de En peligro, Asplenium trilobum como Vulnerable además de Citronella mucronata y Polystichum subintegerrimum como especies Casi amenazada. Las otras 14 especies han sido clasificadas en la categoría de Preocupación menor (Tabla 3).

TABLA 3. Categorías de conservación para las especies presentes en la Estación de Biología Terrestre de Hualpén. / Conservation categories for species present in the Hualpén Terrestrial Biology Station.

\begin{tabular}{lc}
\hline ESPECIE & CATEGORÍA DE CONSERVACIÓN \\
\hline Adiantum chilense & Preocupación menor \\
Adiantum sulphureum & Preocupación menor \\
Alstroemeria hookeri & Preocupación menor \\
Asplenium dareoides & Preocupación menor \\
Asplenium trilobum & Vulnerable \\
& Preocupación menor ${ }^{2}$ \\
Blechnum chilense & Preocupación menor \\
Blechnum hastatum & Preocupación menor \\
Citronella mucronata & Casi amenazada \\
Gomortega keule & En peligro \\
Hymenophyllum caudiculatum & Preocupación menor \\
Hymenophyllum plicatum & Preocupación menor \\
Hypolepis poeppigii & Preocupación menor \\
Megalastrum spectabile & Preocupación menor \\
Myrceugenia leptospermoides & Preocupación menor \\
Pitavia punctata & En peligro \\
Pleopeltis macrocarpa & Preocupación menor \\
Polystichum subintegerrimum & Casi amenazada \\
Pteris chilensis & Preocupación menor \\
Puya chilensis & Preocupación menor \\
\hline
\end{tabular}

${ }^{1}$ En las regiones de Biobío y Maule. / In Biobío and Maule regions.

${ }^{2}$ Desde la Región de la Araucanía al sur. / From Araucanía Región to the south. 
Flora vascular de un remanente de bosque esclerófilo costero: MORENO-CHACÓN, M. ET AL.

TABLA 4. Listado de la flora vascular de la Estación de Biología Terrestre de Hualpén ${ }^{1}$.Origen: Origen geográfico. / List of vascular flora of the Hualpén Terrestrial Biology Station ${ }^{1}$. Origen: Geographic origen.

\begin{tabular}{|c|c|c|c|c|}
\hline Clase & FAMILIA & ESPECIES & ORIGEN & НА́вıто \\
\hline \multirow[t]{20}{*}{ Polypodiopsida } & \multirow[t]{2}{*}{ Aspleniaceae } & Asplenium dareoides Desv. & Nativo & Hierba perenne epífita \\
\hline & & Asplenium trilobum Cav. & Nativo & Hierba perenne epífita \\
\hline & Azollaceae & Azolla filiculoides Lam. & Nativo & Hierba anual acuática \\
\hline & \multirow[t]{4}{*}{ Blechnaceae } & Blechnum chilense (Kaulf.) Mett. & Nativo & Hierba perenne \\
\hline & & Blechnum hastatum Kaulf. & Nativo & Hierba perenne \\
\hline & & Blechnum magellanicum (Desv.) Mett. & Nativo & Hierba perenne \\
\hline & & Blechnum mochaenum G. Kunkel & Nativo & Hierba perenne \\
\hline & Dennstaedtiaceae & Hypolepis poeppigii (Kunze) R.A. Rodr. & Nativo & Hierba perenne \\
\hline & \multirow[t]{3}{*}{ Dryopteridaceae } & $\begin{array}{l}\text { Megalastrum spectabile (Kaulf.) A.R. Sm. \& } \\
\text { R.C. Moran }\end{array}$ & Nativo & Hierba perenne \\
\hline & & Polystichum chilense (H. Christ) Diels & Nativo & Hierba perenne \\
\hline & & $\begin{array}{l}\text { Polystichum subintegerrimum (Hook. \& Arn.) } \\
\text { R.A. Rodr. }\end{array}$ & Endémico & Hierba perenne \\
\hline & Equisetaceae & Equisetum bogotense Kunth & Nativo & Hierba perenne \\
\hline & \multirow[t]{3}{*}{ Hymenophyllaceae } & Hymenophyllum caudiculatum Mart. & Endémico & Hierba perenne epífita \\
\hline & & Hymenophyllum peltatum (Poir.) Desv. & Nativo & Hierba perenne epífita \\
\hline & & Hymenophyllum plicatum Kaulf. & Nativo & Hierba perenne epífita \\
\hline & \multirow[t]{2}{*}{ Polypodiaceae } & Pleopeltis macrocarpa (Bory ex Willd.) Kaulf. & Nativo & Hierba perenne epífita \\
\hline & & Synammia feuillei (Bertero) Copel. & Nativo & Hierba perenne epífita \\
\hline & \multirow[t]{3}{*}{ Pteridaceae } & Adiantum chilense Kaulf. & Nativo & Hierba perenne \\
\hline & & Adiantum sulphureum Kaulf. & Nativo & Hierba perenne \\
\hline & & Pteris chilensis Desv. & Endémico & Hierba perenne \\
\hline \multirow[t]{19}{*}{ Magnoliopsida } & Acanthaceae & Stenandrium dulce (Cav.) Nees & Nativo & Hierba perenne \\
\hline & Aextoxicaceae & Aextoxicon punctatum Ruiz \& Pav. & Nativo & Árbol \\
\hline & Anacardiaceae & Lithrea caustica (Molina) Hook. \& Arn. & Endémico & Árbol \\
\hline & \multirow[t]{8}{*}{ Apiaceae } & Asteriscium chilense Cham. \& Schltdl. & Nativo & Hierba perenne \\
\hline & & Centella asiatica (L.) Urb. & Introducido & Hierba perenne \\
\hline & & Conium maculatum L. & Introducido & Hierba anual o bienal \\
\hline & & Daucus carota L. & Introducido & Hierba anual o bienal \\
\hline & & $\begin{array}{l}\text { Eryngium paniculatum Cav. \& Dombey ex F. } \\
\text { Delaroche }\end{array}$ & Nativo & Hierba perenne \\
\hline & & Foeniculum vulgare Mill. & Introducido & Hierba perenne \\
\hline & & Osmorhiza chilensis Hook. \& Arn. & Nativo & Hierba perenne \\
\hline & & Sanicula crassicaulis Poepp. ex DC. & Nativo & Hierba perenne \\
\hline & \multirow[t]{2}{*}{ Apocynaceae } & Elytropus chilensis (A. DC.) Müll. Arg. & Nativo & Arbusto trepador \\
\hline & & Vinca major $\mathrm{L}$. & Introducido & Hierba perenne \\
\hline & Araliaceae & Hedera helix $\mathrm{L}$. & Introducido & Arbusto trepador \\
\hline & \multirow{5}{*}{ Asteraceae } & Achillea millefolium $\mathrm{L}$. & Introducido & Hierba perenne \\
\hline & & Acrisione denticulata (Hook. \& Arn.) B. Nord. & Endémico & Arbusto \\
\hline & & Anthemis cotula $\mathrm{L}$. & Introducido & Hierba anual \\
\hline & & Arctotheca calendula (L.) Levyns & Introducido & Hierba anual o perenne \\
\hline & & Baccharis linearis (Ruiz \& Pav.) Pers. & Nativo & Arbusto \\
\hline
\end{tabular}


Gayana Bot. 75(1), 2018

\begin{tabular}{|c|c|c|c|c|}
\hline Clase & FAMILIA & ESPECIES & ORIGEN & HÁBIто \\
\hline & & Baccharis racemosa (Ruiz \& Pav.) DC. & Nativo & Arbusto \\
\hline & & Baccharis vernalis F.H. Hellwig & Endémico & Arbusto \\
\hline & & Bidens laevis (L.) Britton, Stern \& Poggenb. & Nativo & Hierba perenne \\
\hline & & Carduus pycnocephalus L. & Introducido & Hierba anual o bienal \\
\hline & & Centaurea bulbosa Hook. \& Arn. & Nativo & Hierba perenne \\
\hline & & $\begin{array}{l}\text { Centipeda elatinoides (Less.) Benth. \& Hook.f. } \\
\text { ex O. Hoffm. }\end{array}$ & Nativo & Hierba perenne \\
\hline & & Chaetanthera chilensis (Willd.) DC. & Endémico & Hierba perenne \\
\hline & & Chaetanthera $x$ serrata Ruiz \& Pav. & Nativo & Hierba perenne \\
\hline & & Chaptalia exscapa (Pers.) Baker & Nativo & Hierba perenne \\
\hline & & Chevreulia pusilla DC. & Nativo & Hierba perenne \\
\hline & & Cichorium intybus L. & Introducido & Hierba anual o bienal \\
\hline & & Cirsium vulgare (Savi) Ten. & Introducido & Hierba anual o bienal \\
\hline & & Cladanthus mixtus (L.) Chevall. & Introducido & Hierba anual \\
\hline & & Crepis capillaris (L.) Wallr. & Introducido & Hierba anual \\
\hline & & Eupatorium glechonophyllum Less. & Nativo & Arbusto \\
\hline & & Hedypnois rhagadioloides (L.) F.W. Schmidt & Introducido & Hierba anual \\
\hline & & Helenium glaucum (Cav.) Stuntz & Endémico & Hierba perenne \\
\hline & & Hieracium chilense Less. & Nativo & Hierba perenne \\
\hline & & Hypochaeris radicata $\mathrm{L}$. & Introducido & Hierba perenne \\
\hline & & Hypochaeris thrincioides (J. Remy) Reiche & Endémico & Hierba perenne \\
\hline & & Lapsana communis L. & Introducido & Hierba anual \\
\hline & & Leontodon saxatilis Lam. & Introducido & Hierba perenne \\
\hline & & Leucanthemum vulgare Lam. & Introducido & Hierba perenne \\
\hline & & Podanthus ovatifolius Lag. & Nativo & Arbusto \\
\hline & & Proustia pyrifolia DC. & Endémico & Arbusto apoyante \\
\hline & & Pseudognaphalium viravira (Molina) Anderb. & Nativo & Hierba perenne \\
\hline & & Senecio nigrescens Hook. \& Arn. & Nativo & Hierba perenne \\
\hline & & Sonchus asper (L.) Hill & Introducido & Hierba anual o bienal \\
\hline & & Sonchus oleraceus L. & Introducido & Hierba anual \\
\hline & & Taraxacum officinale F.H. Wigg. & Introducido & Hierba perenne \\
\hline & & Triptilion spinosum Ruiz \& Pav. & Endémico & Hierba perenne \\
\hline & Boraginaceae & Cynoglossum creticum Mill. & Introducido & Hierba bienal \\
\hline & & Echium plantagineum $\mathrm{L}$. & Introducido & Hierba anual \\
\hline & & Echium vulgare L. & Introducido & Hierba bienal \\
\hline & & Plagiobothrys polycaulis (Phil.) I.M. Johnst. & Endémico & Hierba anual \\
\hline & Brassicaceae & Brassica rapa $\mathrm{L}$. & Introducido & Hierba anual o bienal \\
\hline & & Capsella bursa-pastoris (L.) Medik. & Introducido & Hierba anual o bienal \\
\hline & & Cardamine bonariensis Pers. & Nativo & Hierba perenne \\
\hline & & Draba verna $\mathrm{L}$. & Introducido & Hierba anual \\
\hline & & Raphanus sativus L. & Introducido & Hierba anual o bienal \\
\hline & Calceolariaceae & Calceolaria corymbosa Ruiz \& Pav. & Endémico & Hierba perenne \\
\hline & & Calceolaria integrifolia $\mathrm{L}$. & Endémico & Arbusto \\
\hline
\end{tabular}


Flora vascular de un remanente de bosque esclerófilo costero: MORENO-CHACÓN, M. ET AL.

\begin{tabular}{|c|c|c|c|c|}
\hline Clase & FAMILIA & EsPECIES & ORIGEN & HÁBITo \\
\hline & & Jovellana violacea (Cav.) G. Don & Endémico & Arbusto \\
\hline & Callitrichaceae & Callitriche lechleri (Hegelm.) Fassett & Nativo & Hierba anual acuática \\
\hline & Campanulaceae & Lobelia tupa $\mathrm{L}$. & Nativo & Hierba perenne \\
\hline & & Wahlenbergia linarioides (Lam.) A. DC. & Nativo & Hierba perenne \\
\hline & Cardiopteridaceae & Citronella mucronata (Ruiz \& Pav.) D. Don & Endémico & Árbol \\
\hline & Caryophyllaceae & Cerastium glomeratum Thuill. & Introducido & Hierba anual \\
\hline & & Paronychia chilensis DC. & Nativo & Hierba perenne \\
\hline & & Petrorhagia dubia (Raf.) G. López \& Romo & Introducido & Hierba anual \\
\hline & & Polycarpon tetraphyllum (L.) L. & Introducido & Hierba anual \\
\hline & & Silene gallica $\mathrm{L}$. & Introducido & Hierba anual \\
\hline & & Stellaria arvalis Fenzl ex F. Phil. & Endémico & Hierba perenne \\
\hline & & Stellaria chilensis Pedersen & Nativo & Hierba perenne \\
\hline & & Stellaria media (L.) Cirillo & Introducido & Hierba anual \\
\hline & Celastraceae & Maytenus boaria Molina & Nativo & Árbol \\
\hline & Chenopodiaceae & Chenopodium album $\mathrm{L}$. & Introducido & Hierba anual \\
\hline & Convolvulaceae & Convolvulus arvensis L. & Introducido & Hierba perenne trepadora \\
\hline & & Convolvulus hermanniae L'Hér. & Nativo & Hierba perenne \\
\hline & & Cuscuta chilensis Ker Gawl. & Nativo & Hierba anual parásita \\
\hline & & Dichondra sericea $\mathrm{Sw}$. & Nativo & Hierba perenne \\
\hline & Cunoniaceae & Eucryphia cordifolia Cav. & Nativo & Árbol \\
\hline & Dipsacaceae & Dipsacus sativus (L.) Honck. & Introducido & Hierba bienal \\
\hline & Elaeocarpaceae & Aristotelia chilensis (Molina) Stuntz & Nativo & Árbol \\
\hline & Ericaceae & Gaultheria insana (Molina) D.J. Middleton & Nativo & Arbusto \\
\hline & Escalloniaceae & Escallonia pulverulenta (Ruiz \& Pav.) Pers. & Endémico & Arbusto o árbol \\
\hline & Euphorbiaceae & Chiropetalum tricuspidatum (Lam.) A. Juss. & Endémico & Subarbusto \\
\hline & & Euphorbia peplus L. & Introducido & Hierba anual \\
\hline & Fabaceae & Galega officinalis L. & Introducido & Hierba perenne \\
\hline & & Genista monspessulana (L.) L.A.S. Johnson & Introducido & Arbusto \\
\hline & & Lathyrus magellanicus Lam. & Nativo & Hierba perenne trepadora \\
\hline & & Lotus pedunculatus Cav. & Introducido & Hierba perenne \\
\hline & & Lotus subpinnatus Lag. & Endémico & Hierba anual \\
\hline & & Lupinus arboreus Sims & Introducido & Arbusto \\
\hline & & Lupinus microcarpus Sims & Nativo & Hierba anual \\
\hline & & Medicago arabica (L.) Huds. & Introducido & Hierba anual \\
\hline & & Medicago polymorpha $\mathrm{L}$. & Introducido & Hierba anual \\
\hline & & Ornithopus compressus L. & Introducido & Hierba anual \\
\hline & & Senna stipulacea (Aiton) H.S. Irwin \& Barneby & Endémico & Arbusto \\
\hline & & Sophora cassioides (Phil.) Sparre & Endémico & Árbol \\
\hline & & Trifolium dubium Sibth. & Introducido & Hierba anual \\
\hline & & Trifolium polymorphum Poir. & Nativo & Hierba perenne \\
\hline & & Ulex europaeus L. & Introducido & Arbusto \\
\hline & & Vicia benghalensis L. & Introducido & Hierba anual \\
\hline & & Vicia hirsuta (L.) Gray & Introducido & Hierba anual \\
\hline
\end{tabular}




\begin{tabular}{|c|c|c|c|c|}
\hline Clase & FAMILIA & ESPECIES & ORIGEN & НÁBITO \\
\hline & & Vicia sativa L. subsp. nigra (L.) Ehrh. & Introducido & Hierba anual \\
\hline & & Vicia sativa L. subsp. sativa & Introducido & Hierba anual \\
\hline & Francoaceae & Francoa appendiculata Cav. & Endémico & Hierba perenne \\
\hline & \multirow[t]{4}{*}{ Geraniaceae } & Erodium cicutarium (L.) L'Hér. ex Aiton & Introducido & Hierba anual o bienal \\
\hline & & Geranium berteroanum Colla & Nativo & Hierba perenne \\
\hline & & Geranium core-core Steud. & Nativo & Hierba perenne \\
\hline & & Geranium purpureum Vill. & Introducido & Hierba anual \\
\hline & Gesneriaceae & $\begin{array}{l}\text { Sarmienta scandens (J.D. Brandis ex Molina) } \\
\text { Pers. }\end{array}$ & Endémico & Subarbusto epifito \\
\hline & Gomortegaceae & Gomortega keule (Molina) Baill. & Endémico & Árbol \\
\hline & Griseliniaceae & Griselinia scandens (Ruiz \& Pav.) Taub. & Endémico & Arbusto \\
\hline & Grossulariaceae & Ribes punctatum Ruiz \& Pav. & Nativo & Arbusto \\
\hline & Haloragaceae & Myriophyllum aquaticum (Vell.) Verdc. & Nativo & Hierba perenne acuática \\
\hline & \multirow[t]{2}{*}{ Hypericaceae } & Hypericum caespitosum Cham. \& Schltdl. & Endémico & Hierba anual o perenne \\
\hline & & Hypericum perforatum L. & Introducido & Hierba perenne \\
\hline & \multirow[t]{4}{*}{ Lamiaceae } & Melissa officinalis L. & Introducido & Hierba perenne \\
\hline & & Mentha pulegium $\mathrm{L}$. & Introducido & Hierba perenne \\
\hline & & Prunella vulgaris L. & Introducido & Hierba perenne \\
\hline & & Teucrium bicolor $\mathrm{Sm}$. & Endémico & Arbusto \\
\hline & \multirow[t]{2}{*}{ Lardizabalaceae } & Boquila trifoliolata (DC.) Decne. & Nativo & Arbusto trepador \\
\hline & & Lardizabala biternata Ruiz \& Pav. & Endémico & Arbusto trepador \\
\hline & Lauraceae & Cryptocarya alba (Molina) Looser & Endémico & Árbol \\
\hline & \multirow[t]{3}{*}{ Linaceae } & $\begin{array}{l}\text { Cliococca selaginoides (Lam.) C.M. Rogers \& } \\
\text { Mildner }\end{array}$ & Nativo & Hierba perenne \\
\hline & & Linum bienne Mill. & Introducido & Hierba perenne \\
\hline & & Linum chamissonis Schiede & Nativo & Hierba perenne \\
\hline & \multirow[t]{2}{*}{ Loasaceae } & Loasa acanthifolia Desr. & Nativo & Hierba perenne \\
\hline & & Loasa triloba Dombey ex Juss. & Nativo & Hierba anual \\
\hline & \multirow[t]{2}{*}{ Loranthaceae } & Notanthera heterophylla Ruiz \& Pav. (G. Don) & Endémico & Arbusto parásito \\
\hline & & Tristerix corymbosus (L.) Kuijt & Nativo & Arbusto parásito \\
\hline & Lythraceae & Lythrum hyssopifolium L. & Introducido & Hierba anual \\
\hline & Malvaceae & Modiola caroliniana (L.) G. Don & Introducido & Hierba perenne \\
\hline & Monimiaceae & Peumus boldus Molina & Endémico & Árbol \\
\hline & \multirow[t]{9}{*}{ Myrtaceae } & $\begin{array}{l}\text { Amomyrtus luma (Molina) D. Legrand \& } \\
\text { Kausel }\end{array}$ & Nativo & Árbol \\
\hline & & $\begin{array}{l}\text { Blepharocalyx cruckshanksii (Hook. \& Arn.) } \\
\text { Nied. }\end{array}$ & Endémico & Árbol \\
\hline & & Luma apiculata (DC.) Burret & Nativo & Árbol \\
\hline & & Myrceugenia exsucca (DC.) O. Berg & Nativo & Árbol \\
\hline & & Myrceugenia leptospermoides (DC.) Kausel & Endémico & Arbusto \\
\hline & & Myrceugenia obtusa (DC.) O. Berg & Endémico & Arbusto o árbol \\
\hline & & Myrceugenia parvifolia (DC.) Kausel & Endémico & Arbusto o árbol \\
\hline & & Myrceugenia planipes (Hook. \& Arn.) O. Berg & Nativo & Árbol \\
\hline & & Ugni molinae Turcz. & Nativo & Arbusto \\
\hline
\end{tabular}


Flora vascular de un remanente de bosque esclerófilo costero: MORENO-CHACÓN, M. ET AL.

\begin{tabular}{|c|c|c|c|c|}
\hline Clase & FAMILIA & ESPECIES & ORIGEN & НÁBITO \\
\hline & \multirow[t]{5}{*}{ Onagraceae } & Clarkia tenella (Cav.) H.F. Lewis \& M.R. Lewis & Nativo & Hierba anual \\
\hline & & Fuchsia magellanica Lam. & Nativo & Arbusto \\
\hline & & Ludwigia peploides (Kunth) P.H. Raven & Nativo & Hierba perenne acuática \\
\hline & & Oenothera acaulis Cav. & Endémico & Hierba perenne \\
\hline & & Oenothera stricta Ledeb. ex Link & Nativo & Hierba anual o bienal \\
\hline & \multirow[t]{2}{*}{ Orobanchaceae } & Bartsia trixago L. & Introducido & Hierba anual \\
\hline & & Euphrasia meiantha $\mathrm{Clos}$ & Nativo & Hierba anual \\
\hline & \multirow[t]{4}{*}{ Oxalidaceae } & Oxalis gyrorhiza Bertero ex Colla & Endémico & Hierba perenne \\
\hline & & Oxalis micrantha Bertero ex Savi & Nativo & Hierba anual \\
\hline & & Oxalis perdicaria (Molina) Bertero & Nativo & Hierba perenne \\
\hline & & Oxalis rosea Jacq. & Endémico & Hierba anual \\
\hline & Papaveraceae & Fumaria capreolata $\mathrm{L}$. & Introducido & Hierba anual \\
\hline & Phytolaccaceae & Ercilla spicata (Bertero) Moq. & Endémico & Arbusto trepador \\
\hline & \multirow[t]{3}{*}{ Plantaginaceae } & Fonkia uliginosa Phil. & Nativo & Hierba perenne \\
\hline & & Plantago lanceolata $\mathrm{L}$. & Introducido & Hierba perenne \\
\hline & & Plantago truncata Cham. \& Schltdl. & Endémico & Hierba perenne \\
\hline & Polygalaceae & Polygala gayi A.W. Benn. & Nativo & Hierba anual o bienal \\
\hline & \multirow[t]{8}{*}{ Polygonaceae } & Muehlenbeckia hastulata (Sm.) I.M. Johnst. & Nativo & Arbusto trepador \\
\hline & & Polygonum hydropiperoides Michx. & Nativo & Hierba perenne \\
\hline & & Polygonum persicaria $\mathrm{L}$. & Introducido & Hierba anual \\
\hline & & Rumex acetosella $\mathrm{L}$. & Introducido & Hierba perenne \\
\hline & & Rumex conglomeratus Murray & Introducido & Hierba perenne \\
\hline & & Rumex crispus L. & Introducido & Hierba perenne \\
\hline & & Rumex maricola J. Remy & Endémico & Hierba perenne \\
\hline & & Rumex pulcher L. & Introducido & Hierba perenne \\
\hline & \multirow[t]{2}{*}{ Primulaceae } & Anagallis alternifolia Cav. & Nativo & Hierba perenne \\
\hline & & Anagallis arvensis L. & Introducido & Hierba anual \\
\hline & \multirow[t]{2}{*}{ Proteaceae } & Gevuina avellana Molina & Nativo & Árbol \\
\hline & & Lomatia dentata (Ruiz \& Pav.) R. Br. & Nativo & Árbol \\
\hline & Ranunculaceae & Ranunculus muricatus L. & Introducido & Hierba anual \\
\hline & Rhamnaceae & Rhamnus diffusus Clos & Nativo & Arbusto \\
\hline & \multirow[t]{4}{*}{ Rosaceae } & Kageneckia oblonga Ruiz \& Pav. & Endémico & Árbol \\
\hline & & Rosa rubiginosa $\mathrm{L}$. & Introducido & Arbusto \\
\hline & & Rubus ulmifolius Schott & Introducido & Arbusto \\
\hline & & Sanguisorba minor Scop. & Introducido & Hierba perenne \\
\hline & \multirow[t]{5}{*}{ Rubiaceae } & Galium aparine $\mathrm{L}$. & Introducido & Hierba anual \\
\hline & & Galium hypocarpium (L.) Endl. ex Griseb. & Nativo & Hierba perenne \\
\hline & & Nertera granadensis (Mutis ex L.f.) Druce & Nativo & Hierba perenne \\
\hline & & Oldenlandia salzmannii (DC.) Benth. \& Hook. & Nativo & Hierba anual \\
\hline & & Sherardia arvensis L. & Introducido & Hierba anual \\
\hline & Rutaceae & Pitavia punctata (Ruiz \& Pav.) Molina & Endémico & Árbol \\
\hline & \multirow[t]{2}{*}{ Salicaceae } & Azara integrifolia Ruiz \& Pav. & Endémico & Arbusto \\
\hline & & Azara serrata Ruiz \& Pav. & Endémico & Arbusto \\
\hline
\end{tabular}




\begin{tabular}{|c|c|c|c|c|}
\hline Clase & FAMILIA & ESPECIES & ORIGEN & HÁBIто \\
\hline & & Salix babylonica L. & Introducido & Árbol \\
\hline & & Salix caprea $\mathrm{L}$. & Introducido & Arbusto \\
\hline & Schoepfiaceae & Quinchamalium chilense Molina & Nativo & Hierba perenne \\
\hline & Scrophulariaceae & Alonsoa meridionalis (L.f.) Kuntze & Nativo & Hierba perenne \\
\hline & & Verbascum thapsus L. & Introducido & Hierba bienal \\
\hline & & Verbascum virgatum Stokes & Introducido & Hierba bienal \\
\hline & Solanaceae & Cestrum parqui L’Hér. & Nativo & Arbusto \\
\hline & & Nierembergia repens Ruiz \& Pav. & Nativo & Hierba perenne \\
\hline & & Solanum crispum Ruiz \& Pav. & Nativo & Arbusto \\
\hline & & Solanum nigrum L. & Introducido & Hierba anual \\
\hline & Tropaeolaceae & Tropaeolum ciliatum Ruiz \& Pav. & Endémico & Hierba perenne trepadora \\
\hline & Verbenaceae & Glandularia laciniata (L.) Schnack \& Covas & Nativo & Hierba perenne \\
\hline & & Rhaphithamnus spinosus (Juss.) Moldenke & Nativo & Árbol \\
\hline & & Verbena litoralis Kunth & Nativo & Hierba perenne \\
\hline & Violaceae & $\begin{array}{l}\text { Pombalia parviflora (Mutis ex L.f.) Paula- } \\
\text { Souza }\end{array}$ & Nativo & Hierba anual o perenne \\
\hline & & Viola capillaris Pers. & Nativo & Subarbusto \\
\hline & & Viola huidobrii Gay & Nativo & Hierba perenne \\
\hline & & Viola maculata Cav. & Nativo & Hierba perenne \\
\hline & & Viola reichei Skottsb & Nativo & Hierba perenne \\
\hline & Vitaceae & Cissus striata Ruiz \& Pav. & Nativo & Arbusto trepador \\
\hline & Winteraceae & Drimys winteri J.R. Forst. \& G. Forst. & Nativo & Árbol \\
\hline \multirow[t]{20}{*}{ Liliopsida } & Alstroemeriaceae & Alstroemeria hookeri Lodd subsp. hookeri & Endémico & Hierba perenne \\
\hline & & Alstroemeria ligtu L. subsp. ligtu & Endémico & Hierba perenne \\
\hline & & Bomarea salsilla (L.) Herb. & Endémico & Hierba perenne trepadora \\
\hline & Amaryllidaceae & Rhodophiala maculata (L'Hér.) Ravenna & Nativo & Hierba perenne \\
\hline & Asparagaceae & Oziroë biflora (Ruiz \& Pav.) Speta & Nativo & Hierba perenne \\
\hline & & $\begin{array}{l}\text { Trichopetalum plumosum (Ruiz \& Pav.) J.F. } \\
\text { Macbr. }\end{array}$ & Endémico & Hierba perenne \\
\hline & Bromeliaceae & Fascicularia bicolor (Ruiz \& Pav.) Mez & Endémico & Hierba perenne epífita \\
\hline & & Greigia sphacelata (Ruiz \& Pav.) Regel & Endémico & Hierba perenne \\
\hline & & Puya chilensis Molina & Endémico & Hierba perenne \\
\hline & Corsiaceae & Arachnitis uniflora Phil. & Nativo & Hierba perenne \\
\hline & Cyperaceae & Carex brongniartii Kunth & Nativo & Hierba perenne \\
\hline & & Carex polysticha Boeck. & Nativo & Hierba perenne \\
\hline & & Cyperus eragrostis Lam. & Nativo & Hierba perenne \\
\hline & & Cyperus odoratus L. & Nativo & Hierba perenne palustre \\
\hline & & Uncinia multifaria Nees ex Boott & Endémico & Hierba perenne \\
\hline & & Uncinia phleoides (Cav.) Pers. & Nativo & Hierba perenne \\
\hline & Dioscoreaceae & Dioscorea pedicellata Phil. & Endémico & Hierba perenne trepadora \\
\hline & Hemerocallidaceae & Pasithea caerulea (Ruiz \& Pav.) D. Don & Nativo & Hierba perenne \\
\hline & Iridaceae & Herbertia lahue (Molina) Goldblatt & Nativo & Hierba perenne \\
\hline & & Libertia chilensis (Molina) Gunckel & Nativo & Hierba perenne \\
\hline
\end{tabular}


Flora vascular de un remanente de bosque esclerófilo costero: MORENO-CHACÓN, M. ET AL.

\begin{tabular}{|c|c|c|c|c|}
\hline Clase & FAMILIA & ESPECIES & ORIGEN & НА́вІто \\
\hline & \multirow{8}{*}{ Juncaceae } & Libertia sessiliflora (Poepp.) Skottsb. & Endémico & Hierba perenne \\
\hline & & Sisyrinchium chilense Hook. & Nativo & Hierba perenne \\
\hline & & Sisyrinchium graminifolium Lindl. & Nativo & Hierba perenne \\
\hline & & $\begin{array}{l}\text { Solenomelus pedunculatus (Gillies ex Hook.) } \\
\text { Hochr. }\end{array}$ & Endémico & Hierba perenne \\
\hline & & Juncus bufonius L. & Nativo & Hierba anual \\
\hline & & Juncus imbricatus Laharpe & Nativo & Hierba perenne \\
\hline & & Juncus involucratus Steud ex Buchenau & Nativo & Hierba perenne \\
\hline & & Juncus procerus E. Мey. & Nativo & Hierba perenne \\
\hline & Luzuriagaceae & Luzuriaga radicans Ruiz \& Pav. & Nativo & Subarbusto trepador \\
\hline & \multirow[t]{4}{*}{ Orchidaceae } & Brachystele unilateralis (Poir.) Schltr. & Introducido & Hierba perenne \\
\hline & & Chloraea barbata Lindl. & Nativo & Hierba perenne \\
\hline & & Chloraea gavilu Lindl. & Endémico & Hierba perenne \\
\hline & & $\begin{array}{l}\text { Gavilea longibracteata (Lindl.) Sparre ex L.E. } \\
\text { Navas }\end{array}$ & Endémico & Hierba perenne \\
\hline & Philesiaceae & Lapageria rosea Ruiz \& Pav. & Endémico & Arbusto trepador \\
\hline & \multirow[t]{26}{*}{ Poaceae } & Avena barbata Pott ex Link & Introducido & Hierba anual \\
\hline & & Agrostis capillaris L. & Introducido & Hierba perenne \\
\hline & & Agrostis castellana Boiss. \& Reut. & Introducido & Hierba perenne \\
\hline & & Aira caryophyllea L. & Introducido & Hierba anual \\
\hline & & $\begin{array}{l}\text { Arrhenatherum elatius (L.) P. Beauv. ex J. Presl } \\
\& \text { C. Presl var. bulbosum (Willd.) Spenn. }\end{array}$ & Introducido & Hierba perenne \\
\hline & & Avena fatua $\mathrm{L}$. & Introducido & Hierba anual \\
\hline & & Briza maxima L. & Introducido & Hierba anual \\
\hline & & Briza minor L. & Introducido & Hierba anual \\
\hline & & Bromus hordeaceus L. & Introducido & Hierba anual \\
\hline & & $\begin{array}{l}\text { Chascolytrum koelerioides (Trin.) Essi, Longhi- } \\
\text { Wagner \& Souza-Chies }\end{array}$ & Endémico & Hierba perenne \\
\hline & & Chascolytrum subaristatum (Lam.) Desv. & Nativo & Hierba perenne \\
\hline & & Chusquea quila Kunth & Endémico & Hierba subleñosa perenne \\
\hline & & Cynosurus echinatus L. & Introducido & Hierba anual \\
\hline & & Dactylis glomerata L. & Introducido & Hierba perenne \\
\hline & & Festuca arundinacea Schreb. & Introducido & Hierba perenne \\
\hline & & Holcus lanatus L. & Introducido & Hierba anual \\
\hline & & Hordeum murinum L. & Introducido & Hierba anual \\
\hline & & Lagurus ovatus L. & Introducido & Hierba anual \\
\hline & & Lolium multiflorum Lam. & Introducido & Hierba anual o bienal \\
\hline & & Melica violaceae Cav. & Endémico & Hierba perenne \\
\hline & & Nassella duriuscula (Phil.) Barkworth & Endémico & Hierba perenne \\
\hline & & Nassella gigantea (Steud.) M. Muñoz & Nativo & Hierba perenne \\
\hline & & Nassella laevissima (Phil.) Barkworth & Nativo & Hierba perenne \\
\hline & & Paspalum dasypleurum Kunze ex E. Desv. & Endémico & Hierba perenne \\
\hline & & Piptochaetium angolense Phil. & Endémico & Hierba perenne \\
\hline & & Piptochaetium depressum (Steud.) C. Peña & Endémico & Hierba perenne \\
\hline
\end{tabular}




\begin{tabular}{|c|c|c|c|c|}
\hline Clase & FAMILIA & EsPeCIES & ORIGEN & НÁBIто \\
\hline & & $\begin{array}{l}\text { Piptochaetium fuscum (Nees ex Steud.) } \\
\text { Barkworth, Ciald. \& Gandhi }\end{array}$ & Endémico & Hierba perenne \\
\hline & & Poa annua $\mathrm{L}$. & Introducido & Hierba anual \\
\hline & & Poa denudata Steud. & Endémico & Hierba perenne \\
\hline & & Poa pratensis L. & Introducido & Hierba perenne \\
\hline & & Polypogon viridis (Gouan) Breistr. & Introducido & Hierba perenne \\
\hline & & Trisetum caudulatum Trin. & Nativo & Hierba perenne \\
\hline & Tecophilaeaceae & Conanthera bifolia Ruiz \& Pav. & Endémico & Hierba perenne \\
\hline
\end{tabular}

${ }^{1}$ Nomenclatura de acuerdo al Catálogo de las Plantas Vasculares del Cono Sur/ Nomenclature according to Catalogue of the Vascular Plants of the Southern Cone.

\section{DISCUSIÓN}

El listado de especies de la flora vascular obtenido muestra que la EBT posee una alta riqueza de plantas vasculares, a pesar de ser un polígono de superficie reducida (70 ha) dentro del Santuario de la Naturaleza de la Península de Hualpén que comprende 2.662 ha. En esta área se determinaron 195 especies nativas y endémicas, lo que equivale al $14 \%$ de la riqueza total descrita para la Región del Biobío. El alto número de especies encontradas en la EBT coincide con lo encontrado en el trabajo de Teneb et al. (2004), donde varios corotipos de árboles y arbustos presentes en la Región del Biobío se encuentran en la zona costera que incluye la Península de Hualpén. Al mismo tiempo, el número de especies, familias y géneros de la EBT es similar a los datos de diversidad existentes para la Cordillera de la Costa de la Región del Biobío (Cavieres et al. 2005). Estos antecedentes permiten evidenciar que la EBT es un remanente de bosque costero que, a pesar de su aislamiento actual y alto grado de degradación antrópica de las áreas aledañas, ocupadas principalmente por plantaciones forestales (Cavieres et al. 2005, Heilmayr et al. 2016), permite conservar la diversidad a nivel local.

Si consideramos la identidad de las especies encontramos que dentro de la EBT se hallan 17 de las 26 especies potenciales descritas en la composición característica del bosque esclerófilo mediterráneo costero de Lithrea caustica y Azara integrifolia (Luebert \& Pliscoff 2006). Entre estas especies se encuentran Azara integrifolia (Salicaceae), Blechnum hastatum (Blechnaceae), Cryptocarya alba (Lauraceae), Lardizabala biternata (Lardizabalaceae), Lithrea caustica (Anacardiaceae), Muehlenbeckia hastulata (Polygonaceae) y Ugni molinae (Myrtaceae). De lo anterior podemos confirmar que no solo hay un alto grado de riqueza en la EBT, sino que además conserva componentes florísticos remanentes de la vegetación original. Nuestro resultado cobra relevancia ya que es un parámetro cuantitativo que no deja de ser significativo al momento de la toma de decisiones en el manejo y conservación de la flora local.
Además del alto número de especies y la mantención de elementos originales del bosque esclerófilo costero de la zona, la EBT alberga poblaciones de 5 especies que se encuentran clasificadas en alguna de las categorías de conservación, destacando el rol de las áreas complementarias al SNASPE, como sitios de protección de especies y hábitats. En la EBT se encuentran ejemplares de Gomortega keule, único representante de la familia Gomortegaceae (Muñoz 1966) y Pitavia punctata especie endémica de Chile (Rutaceae) y única representante del género (Muñoz-Schick et al. 2012). Las dos especies son árboles emblemáticos de la Región del Biobío y se han clasificado en la categoría de En peligro, la que indica que una especie tiene una alta probabilidad de desaparecer en el mediano plazo (MMA 2010). En el caso de G. keule se ha reportado una escasez de plántulas en poblaciones naturales debido a la falta de reclutamiento por semillas en las condiciones actuales del bosque (Villegas et al. 2003, Hechenleitner et al. 2005). En P. punctata la vulnerabilidad de la especie aumenta por la reducción de los simbiontes de raíz, por la escasez de polinizadores y por un aumento en la presión relativa de agentes patógenos como los insectos fitófagos, los hongos y las bacterias (Le Quesne 1995). P. punctata es una especie de la que se conocen pocas poblaciones, en el marco del SNASPE se la protege sólo en las reservas nacionales Los Ruiles y Malleco. Un caso similar es el de G. keule cuyas poblaciones sólo se protegen en la Reserva Nacional Los Queules. Por su parte, Asplenium trilobum (Aspleniaceae), especie de helecho (con hábito de crecimiento tipo epífito obligado) asociado a los bosques húmedos (Rodríguez et al. 2009), se clasifica en la categoría de Vulnerable en las regiones de Maule y Biobío. En esta zona, que coincide con su límite natural de distribución norte (Rodríguez et al. 2009), las poblaciones de $A$. trilobum se encuentran fragmentadas y restringidas a remanentes de bosque nativo. La principal amenaza a estas poblaciones está asociada a los cambios en la vegetación nativa debido a sustitución y tala (Baeza et al. 1998).

Dada la importancia que la EBT presenta en la protección de la riqueza y composición de especies 
nativas y endémicas del remanente del bosque esclerófilo mediterráneo costero, resulta preocupante el alto porcentaje $(33,7 \%)$ de especies introducidas que se encuentran en ella. El número de especies introducidas en la EBT supera los valores encontrados por Cavieres et al. (2005) para zonas cercanas y situadas en la Cordillera de la Costa de la Región del Biobío (entre 49 y 81 especies). Este alto número de especies introducidas es preocupante para la conservación de un área natural, pues se ha descrito que la introducción de especies es una de las principales causas de la pérdida de biodiversidad (Vitousek et al. 1997, Sala et al. 2000). El riesgo potencial para la flora nativa y endémica se asocia a la presencia de especies introducidas naturalizadas, que tienen la capacidad de reproducirse y mantener poblaciones estables (Richardson et al. 2000) así como de invasoras, que causan impactos ecológicos modificando la composición, funcionamiento y estructura del ecosistema nativo (Parker et al. 1999, Fuentes et al. 2014).

De las 99 especies introducidas presentes en la EBT el $13 \%$ corresponde a especies naturalizadas y un $55 \%$ corresponde a especies invasoras (Fuentes et al. 2013, 2014). En la EBT encontramos especies de Fabaceae como Genista monspessulana (L.) y Ulex europaeus L., las cuales favorecen las condiciones para la generación de incendios (Pauchard et al. 2008, Fuentes et al. 2014). Otras especies como Taraxacum officinale (Asteraceae), Echium vulgare (Boraginaceae), Capsella bursa-pastoris (Brassicaceae), Prunella vulgaris (Lamiaceae), Plantago lanceolata L. (Plantaginaceae) y Rosa rubiginosa (Rosaceae), podrían desplazar a las especies nativas, impidiendo su regeneración (Quiroz et al. 2009, Fuentes et al. 2014). Especies como Sonchus asper (Asteraceae) y Convolvulus arvensis (Convolvulaceae) producen compuestos tóxicos que impiden el crecimiento de las especies nativas (Fuentes et al. 2014). Adicionalmente, durante el trabajo de campo observamos que las especies introducidas se mantienen mayormente en las áreas de pastizal que en la zona boscosa. Este patrón de distribución es explicado porque el bosque actuaría como una barrera que previene el ingreso de las especies introducidas (Pauchard \& Alaback 2006), dado que se ha propuesto que las comunidades con mayor riqueza de especies nativas tienen una menor probabilidad de ser invadidas debido a que hay menos espacios o nichos ecológicos disponibles para ser colonizados (Elton 1958, Shea \& Chesson 2002, Meiners \& Cadenasso 2005). Sin embargo, el alto número de especies introducidas en la zona de pastizal es un dato relevante, puesto que en esa zona existen poblaciones de especies nativas que podrían verse perjudicadas por el incremento de las especies introducidas consideradas invasoras. Por estas razones, llevar un registro de las especies introducidas se hace imprescindible al momento de formular y aplicar medidas de conservación de la biodiversidad, pudiendo así prevenir sus efectos sobre la flora nativa y endémica (Fuentes et al. 2014).
Finalmente, los resultados obtenidos en nuestro estudio sugieren que el área complementaria de protección que representa la EBT ha permitido conservar la riqueza y composición de especies nativas y endémicas del bosque esclerófilo mediterráneo costero de la Región del Biobío, a pesar de su pequeña superficie y a estar inmersa en un paisaje altamente alterado por actividad humana. Sin embargo, el alto porcentaje de especies introducidas registradas en este estudio es un factor preocupante en términos de conservación de especies nativas y endémicas distribuidas en la zona de pastizal. A partir de lo registrado se considera necesario sumar, a la información aquí recopilada, investigaciones en el ámbito ecológico que permitan conocer los patrones poblacionales y comunitarios, información que será relevante para formular e implementar acciones de protección y manejo del bosque esclerófilo costero de la Región del Biobío.

\section{AGRADECIMIENTOS}

Se agradece a Patricio López, Ricardo León, Tomás Leiton, Cristóbal Carrasco, Rocío Fernández, Antonio Escandón y Sebastián Muñoz. A los revisores anónimos por sus comentarios, y a los proyectos MM-C (21130186), FONDECYT 1140455 y EDPG LPR-161, Dirección de Postgrado-UdeC.

\section{REFERENCIAS}

Aguayo, M., Pauchard, A., Azócar, G., Parra, O. 2009. Cambio del uso del suelo en el centro sur de Chile a fines del siglo XX. Entendiendo la dinámica espacial y temporal del paisaje. Revista Chilena de Historia Natural 82(3): 361374.

Armesto, J.J., Villagrán, C., Donoso, C. 1994. Desde la era glacial a la industrial: La historia del bosque templado chileno. Ambiente y Desarrollo 10(1): 66-72.

Armesto, J.J., Rozzi, R., Smith-Ramírez, C., Arroyo, M.T.K. 1998. Conservation targets in South American temperate forests. Science 282: 1271-1272.

Arroyo, M.T.K., Matthei, O., Muñoz-Schick, M., Armesto, J.J., Pliscoff, P., Pérez, F., Marticorena, C. 2005. Flora de cuatro Reservas Nacionales en la Cordillera de la Costa de la VII Región (35 $-36^{\circ} \mathrm{S}$ ), Chile, y su papel en la protección de la biodiversidad regional. En: SmithRamírez, C., Armesto, J.J., Valdovinos, C. (eds.), Historia, biodiversidad y ecología de los bosques costeros de Chile, pp. 245-252. Editorial Universitaria. Santiago, Chile.

Baeza, M., Barrera, E., Flores, J., Ramírez, C., Rodríguez, R. 1998. Categorías de Conservación de Pteridophyta Nativas de Chile. Boletín Del Museo Nacional de Historia Natural 47: $23-46$.

Bustamante, R.O., Simonetti, J.A., Grez, A.A., San Martín, J. 2005. Fragmentación y dinámica de regeneración del bosque Maulino: diagnóstico actual y perspectivas futuras. 
En: Smith-Ramírez, C., Armesto, J.J., Valdovinos, C. (eds.), Historia, biodiversidad y ecología de los bosques costeros de Chile, pp. 555-564. Editorial Universitaria, Santiago, Chile.

Carrasco, C. 1976. Flora vascular del Parque Botánico Hualpén. Tesis de pregrado. Instituto de Biología. Universidad de Concepción, Concepción. 267 pp.

Cavieres, L., Minoc, M., Marticorena, A., Marticorena, C., Baeza, C., Arroyo, M.T.K. 2005. Flora vascular de la Cordillera de la Costa en la Región del Biobío: riqueza de especies, géneros, familias y endemismos. En: SmithRamírez, C., Armesto, J.J., Valdovinos, C. (eds.), Historia, biodiversidad y ecología de los bosques costeros de Chile, pp. 245-252. Editorial Universitaria, Santiago, Chile.

CONAF-CONAMA. 1997. Catastro de evaluación de los recursos vegetacionales nativos de Chile. Informe nacional con variables ambientales, CONAMA. Santiago. $90 \mathrm{pp}$.

Donoso, C. 2005. Árboles nativos de Chile. Marisa Cuneo Ediciones, Valdivia. $136 \mathrm{pp}$.

Donoso, C. 2009. Arbustos nativos de Chile. Marisa Cuneo Ediciones, Valdivia. $131 \mathrm{pp}$.

Elton, C. 1958. The Ecology of invasions by animals and plants. Chapman and Hall. London. 181 pp.

Finot, V.L., Marticorena, C., Barrera, J.A. Muñoz-Schick, M., Negritto, M. 2009. Diversidad de la familia Poaceae (Gramineae) en la Región del Bío-Bío, Chile. Gayana Botánica 66(2): 134-157.

FPA. 2016. Guía Santuario de la Naturaleza de la Península de Hualpén. Ministerio del Medio Ambiente. Gobierno de Chile. 75 pp.

Freire, S., Bayón, N.D., Baeza, C., Giuliano, D., Monti, C. 2014. Revision of the genus Pseudognaphalium (Asteraceae, Gnaphalieae) in Chile. Gayana Botánica 71(1): 68-107.

Fuentes, N., Pauchard, A., SÁnchez, P., Esquivel, J., Marticorena, A. 2013. A new comprehensive database of alien plant species in Chile based on herbarium records. Biological Invasions 15(4): 847-858.

Fuentes, N., Sánchez, P., Pauchard, A., Urrutia, A., Cavieres, L., Marticorena. A. 2014. Plantas invasoras del centro-sur de Chile: Una guía de campo. Laboratorio de Invasiones Biológicas (LIB), Concepción. 276 pp.

García, N. 2010. Caracterización de la flora vascular de Altos de Chicauma, Chile (33오). Gayana Botánica 67(1): 65-112.

García, N., OrmazÁbal, C. 2008. Árboles nativos de Chile. Enersis S.A. Santiago. $196 \mathrm{pp}$.

Gómez, P., Hans, S., San Martín, J. 2009. Estructura y composición florística de un matorral bajo plantaciones de Pinus radiata D. Don en Chile central. Gayana Botánica 66(2), 256-268.

Hechenleitner, P., Gardner, M.F., Thomas, P.I., Echeverría, C., Escobar, C., Brownless, P., Martínez, C. 2005. Plantas amenazadas del centro-sur de Chile. Distribución, conservación y propagación. Universidad Austral de Chile y Real Jardín Botánico de Edimburgo. Valdivia.188 pp.

Heilmayr, R., Echeverría, C., Fuentes, R., Lambin, E.F. 2016. A plantation-dominated forest transition in Chile. Applied Geography 75: 71-82.

Hoffmann, A. 1998. Plantas altoandinas en la flora silvestre de Chile. Ediciones Fundación Claudio Gay. Santiago. 281 pp.

Ibarra-Vidal, H., Sepúlveda, C., Saavedra. D., Maldonado, E. 2005. Propuestas de conservación de los bosques nativos en la cordillera del Maule y Biobío (VII y VIII Regiones). En: Smith-Ramírez, C., Armesto, J.J., Valdovinos, C. (eds.), Historia, biodiversidad y ecología de los bosques costeros de Chile, pp. 617-631. Editorial Universitaria, Santiago, Chile.

Le Quesne, C. 1995. Algunos agentes bióticos dañinos asociados a pitao (Pitavia punctata Mol.), Rutaceae endémica en peligro de extinción. CONAF, Santiago. 21 pp.

Luebert, F., Pliscoff, P. 2006. Sinopsis bioclimática y vegetacional de Chile. Editorial Universitaria, Santiago. 310 pp.

Marticorena, C. 1990. Contribución a la estadística de la flora vascular de Chile. Gayana, Botánica 47(2): 85-113.

Marticorena, A., Alarcón, D., Abello, L., Atala, C. 2010. Plantas trepadoras, epífitas y parásitas nativas de Chile. Ediciones Corporación Chilena de la Madera, Concepción. 290 pp.

Marticorena, C., Rodríguez, R. (eds.) 1995. Flora de Chile. Vol. 1. Pteridophyta-Gymnospermae. Universidad de Concepción, Concepción. 351 pp.

Marticorena, C., Rodríguez, R. (eds.) 2001. Flora de Chile. Vol. 2(1). Winteraceae-Ranunculaceae. Universidad de Concepción, Concepción. 99 pp.

Marticorena, C., Rodríguez, R. (eds.) 2003. Flora de Chile. Vol. 2(2): Berberidaceae- Betulaceae. Universidad de Concepción, Concepción. 93 pp.

Marticorena, C., Rodríguez, R. (eds.) 2005. Flora de Chile. Vol. 2(3): Plumbaginaceae- Malvaceae. Universidad de Concepción, Concepción. 128 pp.

Marticorena, C., Rodríguez, R. (eds.) 2011. Flora de Chile. Vol. 3(1): Misodendraceae-Zygophyllaceae. Universidad de Concepción, Concepción. 148 pp.

Matthei, O. 1995. Manual de las malezas que crecen en Chile. Alfabeta Impresores, Santiago. 545 pp.

Meiners, S.J., Cadenasso, M.L. 2005. The relationship between community diversity and exotic plants: cause or consequence of invasion? In: Inderjit (ed.), Invasive plants: ecological and agricultural aspects, pp. 97-114. Birkhäuser Vergal, Basel, Switzerland.

Miranda, A., Altamirano, A., Cayuela, L., Pincheira, F., Lara, A. 2015. Different times, same story: Native forest loss and landscape homogenization in three physiographical areas of south-central of Chile. Applied Geography 60: 20-28.

MMA (Ministerio del Medio Ambiente). 2010. D.S. 29. Reglamento para la clasificación de especies silvestres según estado de conservación. Ministerio del Media Ambiente. Santiago. 9 pp.

Mösbach, E.W. 1992. Botánica indígena de Chile. Museo chileno de arte precolombino, Fundación Andrés Bello, Santiago. $140 \mathrm{pp}$.

Muñoz, C. 1966. Sinopsis de la flora chilena. Editorial Universitaria, Santiago. 840 pp.

Muñoz-Schick, M. 1980. Flora del Parque Nacional Puyehue. Editorial Universitaria, Santiago. 840 pp.

MuÑoz-Schick, M., Moreira-Muñoz, A., EspinozA, S. 2012. Origen del nombre de los géneros de plantas vasculares nativas de Chile y su representatividad en Chile y el mundo. Gayana Botánica 69(2): 309-359.

NiEmeYer, H. 2014. Quantitative screening for alkaloids of native vascular plant species from Chile: biogeographical considerations. Boletín Latinoamericano y del Caribe de Plantas Medicinales y Aromáticas 13(1):109-116. 
Flora vascular de un remanente de bosque esclerófilo costero: Moreno-ChACÓN, M. ET AL.

NovoA, P. 2013. Flora de la Región de Valparaíso. Patrimonios y estado de conservación. Catálogo documentado y fotográfico. Ediciones Fundación Jardín Botánico Nacional, Valparaíso. 360 pp.

Novoa, P., Espejo, J., Alarcón, D., Cisternas, M., Domínguez, E. 2015. Guía de campo de las orquídeas chilenas. Segunda edición. Corporación chilena de la madera, Concepción. $240 \mathrm{pp}$.

Parker, I.M., Simberloff, D.S., Lonsdale, W.M., Goodell, K., Wonham, M., Kareiva, P.M., Willliamson, M.H., Von Holle, B., Moyle, P.B., Byers, J.E., Goldwasser, L. 1999. Impact: toward a framework for understanding the ecological effects of invaders. Biological Invasions 1: 3-19.

Pauchard, A., Alaback, P. 2006. Edge type defines alien plant species invasions along Pinus contorta burned, highway and clearcut forest edges. Forest Ecology and Management 223(1): 327-335.

Pauchard, A., García, R., Peña, E., González, C., Cavieres, L., Bustamante, R.O. 2008. Positive feedbacks between plant invasions and fire regimes: Teline monspesulana (L.) K.Kock (Fabaceae) in central Chile. Biological Invasions 10: 547-553.

Polyméris, C. 1995. Vegetación actual de la Península de Hualpén. Clasificación y dinámica. Tesis de Magíster en Ciencias con mención en Botánica. Facultad de Ciencias Naturales y Oceanográficas. Universidad de Concepción, Concepción. $190 \mathrm{pp}$.

Quintana, V. 1993. Caracterización florística y faunística de un humedal costero de la VIII Región, el caso del Estero Lenga. En: Faranda, F., Parra, O. (eds.), Planificación y gestión de la zona costera, un análisis de caso: Lenga. Proyecto EULA-Chile, pp. 41-56. Serie Propuesta de ordenamiento, Vol. 8, Concepción. Chile.

Quiroz, C.L., Pauchard, A., Marticorena, A., Cavieres, L. 2009. Manual de plantas invasoras del centro-sur de Chile. Laboratorio de Invasiones Biológicas, Universidad de Concepción, Concepción. 45 pp.

Richardson, D.M., Pyšek, P., Rejmánek, M., Barbour, M.G., PanetTA, F.D., West, C.J. 2000. Naturalization and invasion of alien plants: concepts and definitions. Diversity \& Distributions 6: 93-107.

Riedemann, P., Aldunate, G., Teillier, S. 2014. Arbustos nativos de la zona centro-sur de Chile. Guía de campo. Corporación Chilena de la Madera, Concepción. 308 pp.

Rodríguez, R., Grau, J., Baeza, C., Davies, A. 2008a. Lista comentada de las plantas vasculares de los Nevados de Chillán, Chile. Gayana Botánica 65(2): 153-197.

Rodríguez, R., Marticorena, A., Teneb, E. 2008b. Plantas vasculares de los ríos Baker y Pascua, Región de Aysén, Chile. Gayana Botánica 65(1): 39-70.

Rodríguez, R., Dellarosa, V. 1998. Plantas vasculares acuáticas en la Región del Bío-Bío. Universidad de Concepción, Concepción. 38 pp.
Rodríguez, R., Alarcón, D., Espejo, J. 2009. Guía de campo: Helechos nativos del centro y sur de Chile. Ediciones Corporación Chilena de la Madera, Concepción. 212 pp.

Rodríguez, R., Ruiz, E., Elissetche, J.P. 2005. Árboles en Chile. Universidad de Concepción, Concepción. 183 pp.

Sala, O., Chapín, F.S., Armesto, J.J., Berlow, E., Bloomfield, J. 2000. Biodiversity - global biodiversity scenarios for the year 2100. Science 287: 1770-1774.

San Martín, J., Donoso, C. 1995. Estructura florística e impacto antrópico en el bosque maulino de Chile. En: Armesto, J.J., Villagrán, C., Arroyo, M.T.K (eds.), Ecología de los bosques nativos de Chile, pp. 153-168. Editorial Universitaria, Santiago, Chile.

SchulmeYer, D. 1978. Observaciones fitogeográficas sobre la Cordillera de Nahuelbuta. Boletín Informativo IGM II trimestre, Instituto Geográfico Militar, Santiago. 27 pp.

Shea, K., Chesson, P. 2002. Community ecology theory as a framework for biological invasions. Trends in Ecology \& Evolution 17(4): 170-176.

Smith, R., Armesto, J.J. 2002. Importancia biológica de los bosques costeros de la Décima Región: el impacto de la carretera costera sur. Ambiente y Desarrollo 18(1): 6-14.

Smith-Ramírez, C. 2004. The Chilean coastal range: a vanishing center of biodiversity and endemism in southern temperate rain forests. Biodiversity and Conservation 13: 373-393.

Teillier, S., Aldunate, G., Riedemann, P., Niemeyer, H. 2005. Flora de la Reserva Nacional Río Clarillo. Universidad de Chile y CONAF, Santiago. 367 pp.

Teillier, S., Escobar, F. 2013. Revisión del género Gaultheria L. (Ericaceae) en Chile. Gayana Botánica 70(1): 136-153.

Teillier, S., Marticorena, A., Macaya, J., Bonnemaison, C., Delaunoy, J. 2014. Flora de la Reserva Biológica Huilo Huilo, Vol. 1. Fundación Huilo Huilo, Santiago. 473 pp.

Teneb, E.A., Cavieres, L., Parra, M., Marticorena, A. 2004. Patrones geográficos de distribución de árboles y arbustos en la zona de transición climática mediterráneo-templada de Chile. Revista Chilena de Historia Natural 77: 51-71.

Villagrán, C., Armesto, J.J. 2005. Fitogeografía histórica de la Cordillera de la Costa de Chile. En: Smith-Ramírez, C., Armesto, J.J., Valdovinos C. (eds.), Historia, biodiversidad y ecología de los bosques costeros de Chile, pp. 99-115. Editorial Universitaria, Santiago, Chile.

Villegas, D., Le Quesne, C., Lusk, C.H. 2003. Estructura y dinámica de una población de Gomortega keule (Mol.) Baillon en un rodal antiguo de bosque Valdiviano, Cordillera de Nahuelbuta, Chile. Gayana Botánica 60(2): 107-113.

Vitousek, P.M., Mooney, H.A., Lubchenco, J., Melillo, J.M. 1997. Human domination of Earths's ecosystems. Science 277: 494-499.

Zuloaga, F.O., Morrone, O., Belgrano, M.J. (eds.). 2008. Catálogo de las Plantas Vasculares del Cono Sur (Argentina, Sur de Brasil, Chile, Paraguay y Uruguay). Monographs of the Missouri Botanical Garden. 107: 3348 pp.

Recibido: 29.06.2017

Aceptado: 03.05.2018 